DOI: 10.1136/annrheumdis-2017-eular.6490

\section{SAT0209 OBSERVATIONAL STUDY ON THE EFFECTS OF IL-6 INHIBITOR THERAPY ON MYOSTATIN IN PATIENTS WITH RHEUMATOID ARTHRITIS}

M.J. Chapman ${ }^{1}$, R.P. Narayanan ${ }^{2}$, A. Cross $^{3}$, R. Moots ${ }^{3}$, J. Wilding $^{2}$, N. Goodson ${ }^{3} .{ }^{1}$ Department of Rheumatology, Aintree University Hospital; ${ }^{2}$ Department of Obesity and Endocrinology; ${ }^{3}$ Institute of Ageing and Chronic Disease, University of Liverpool, Liverpool, United Kingdom

Background: Rheumatoid cachexia $(\mathrm{RC})$, a phenotype of increased adiposity, reduced lean mass and insulin resistance can occur in rheumatoid arthritis (RA). It is most prevalent in those with high disease activity and is associated with increased cardiovascular morbidity and mortality. Tocilizumab (TCZ), a monoclonal anti-IL-6 antibody, successfully reduces disease activity in RA. It has also been associated with improved insulin sensitivity, increased lean mass and hyperlipidaemia. The serum concentration of myostatin, a myokine released by skeletal muscle, is increased in most disease states associated with cachexia. Moreover, its knockout and inhibition increase lean mass. However, its role in RA has not been fully elucidated.

Objectives: To investigate the effect of tocilizumab treatment on serum myostatin in patients with RA.

Methods: 19 patients with RA (16 female, 3 male) mean age $49.6 y r s$, mean DAS28 6.1, median disease duration 10yrs (range 0 to 30 ), mean BMI 27.4, received $13 \mathrm{IV}$ infusions of TCZ $8 \mathrm{mg} / \mathrm{kg}$ every four weeks as part of a 52 week, single-centre, open-label study (ACT-NEUT [1]). 8 had previously been exposed to a biological agent, 4 were on regular steroids, and all patients received concurrent methotrexate. Serum myostatin was measured at 0,1,3 and 6months of treatment using ELISA. BMI, serum lipid profile, CRP, ESR and DAS28 were measured at each visit $(0,1,3$ and 6 months). Data were analysed using STATA 14. Change between 0,3 and 6 months was analysed using Wilcoxon signed rank test, statistical significance was confirmed using mixed model analysis adjusting for BMI, age and gender. Linear regression with adjustment for age, BMI and gender assessed correlation at baseline.

Results: DAS28, CRP and ESR all significantly decreased with TCZ treatment. A significant increase in BMI (25.5 (IQR 21.6-31.7) vs 26.0 (IQR 21.4-32.5) $\mathrm{p}=0.0052$ ) between baseline and 6 months and serum triglycerides between baseline and 3 months (1.3 (IQR 1.3-2.2) vs 1.6 (IQR 1.3-2.6) p=0.028) was seen with TCZ. Baseline serum myostatin concentrations were negatively correlated with baseline DAS28 $\left(r^{2}=0.39 \mathrm{p}=0.038\right)$. Treatment with tocilizumab for 6 months resulted in a significant increase in serum myostatin from baseline (2.7ng/mL (IQR 2.0-3.1) vs 3.3ng/mL (IQR 3.0-5.0) p <0.001) [Fig. 1]. Moreover, a significant association was seen between baseline myostatin and change in cholesterol at 3 months $\left(r^{2}=0.51 p=0.029\right)$, when adjusting for baseline cholesterol, age, gender, $\mathrm{BMI}$ and change in BMI.

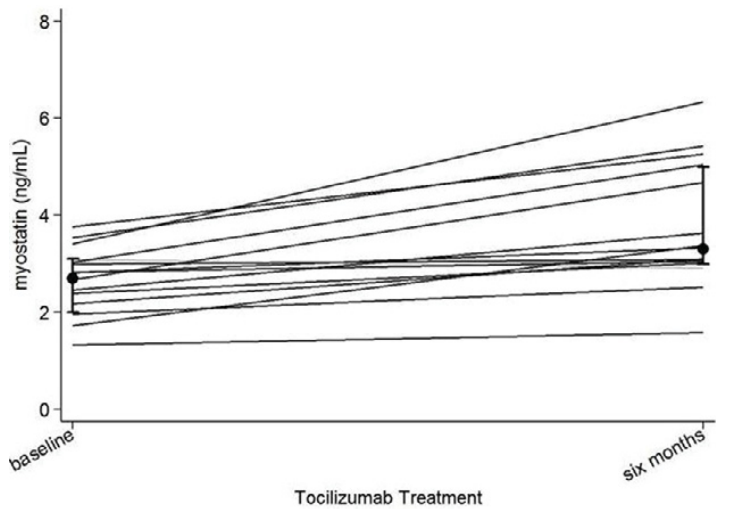

Fig 1. Serum myostatin levels significantly increase after six months of tocilizumab in patients with rheumatoid arthritis. Values shown are actual serum values for individual patients ( $\mathrm{n}=19$ at baseline $\mathrm{n}=15$ at 6 months). Black lines represent increasing serum values, grey lines decreasing values with treatment. Circles represent median $\pm I Q R$. Change evaluated by Wilcoxon signed rank test $\mathrm{p}<0.001$

Conclusions: We have demonstrated a significant correlation between myostatin and DAS28 and a significant change in myostatin with tocilizumab treatment. It is possible that IL- 6 blockade results in a rise in myostatin. This might attenuate any improvement in muscle wasting. Future studies should measure body composition and muscle function to help understand the changes we have observed.

\section{References:}

[1] Wright HL, Cross AL, Edwards SW, Moots RJ. Effects of IL-6 and IL-6 blockade on neutrophil function in vitro and in vivo. Rheumatology. 2014 Jul;53(7):1321-31.

Disclosure of Interest: None declared

DOI: 10.1136/annrheumdis-2017-eular.5831
SAT0210 IMPROVEMENT OF DISEASE ACTIVITY IN A 5-YEAR COHORT OF RHEUMATOID ARTHRITIS PATIENTS TREATED UNDER TREAT TO TARGET RECOMMENDATIONS RECEIVING BIOLOGICAL THERAPY

P. Santos-Moreno ${ }^{1}$, D. Gomez ${ }^{1}$, E. Castillo ${ }^{1}$, R. Giraldo ${ }^{1}$, G. Ballesteros ${ }^{1}$, L. Villarreal ${ }^{2}$, J. Bello ${ }^{3}$, D. Buitrago-Garcia ${ }^{3} .{ }^{1}$ Rheumatology; ${ }^{2}$ Psychology and processes; ${ }^{3}$ Epidemiology, Biomab, Center for Rheumatoid Arthritis, Bogota,

Bogota, Colombia

Background: Treat to Target (T2T) strategy becomes from the need to develop therapeutic targets and tools to achieve defined outcomes in rheumatoid arthritis (RA), this strategy has become recognized as a standard of good practice embodying the principle that rapid attainment of remission, or low disease activity, can halt joint damage and maintain good quality of life. However, there is no direct comparison between biologics in cohorts of patients with long-standing RA using T2T approach in real-life settings, which could have implications in treatment decisions and health economics.

Objectives: The aim of this study was to describe global change in Disease Activity Score 28 (DAS28) using T2T strategy for a 5 year period in patients with biological therapy in a large cohort of patients from a Colombian specialized in RA center with multidisciplinary approach

Methods: A descriptive dynamic cohort study was performed. Records of patients using biological DMARD treatment (Anti-TNF and others) from specialized in RA center were reviewed; those patients were followed-up under T2T standards. Clinical follow-up was according to DAS28 as follows: every $3-5$ weeks (DAS28 $>5.1$ ), every $7-9$ weeks (DAS28 $\geq 3.1$ and $\leq 5.1$ ), and every $11-13$ weeks (DAS28 $<3.1$ ). Therapy had to be adjusted with DAS28 $>3.2$ unless patient's conditions don't permit it. We divided patients in three groups: low disease activity (LDA), moderate disease activity (MDA) and severe disease activity (SDA) patients. Descriptive epidemiology was done, percentages and averages were calculated; the median of each variable was analyzed using t-Student assuming normality for DAS28 distribution and the level activity disease was analyzed using Pearson's statistics.

Results: During 60 month period we included 695 patients with biological therapy, $85 \%$ were female and $15 \%$ male. Mean age was 58 years \pm 11 . At beginning mean DAS28 was $4.1 \pm 1.1$ and $20 \%$ of patients were in SDA, and $55 \%$ in MDA. At the end of 5 year period mean DAS was $3.2 \pm 0.89$ and only $7 \%$ of patients were in SDA and $33 \%$ in MDA. The median was analyzed using t-Student assuming normality for DAS28 distribution. It showed statistical significant improvement $(p<0.00)$

\begin{tabular}{|l|r|r|r|r|}
\hline ACTIVITY LEVEL & TIME 0 n(\%) & $\begin{array}{l}2011-2012 \\
\mathrm{n}(\%)\end{array}$ & $2013-2014(\%)$ & 2015-2016(\%) \\
\hline REM & & $50(7)$ & $166(24)$ & $217(31)$ \\
\hline LDA & $178(26)$ & $236(34)$ & $181(26)$ & $198(28)$ \\
\hline MDA & $382(55)$ & $361(52)$ & $278(40)$ & $234(74)$ \\
\hline SDA & $135(19)$ & $98(14)$ & $70(10)$ & $46(7)$ \\
\hline
\end{tabular}

Conclusions: There was a global improvement of DAS28 in a cohort of RA patients receiving biological therapy followed and treated under recommendations of T2T approach demonstrating that with the strategies mentioned above it is possible to obtain a good control of the activity of the disease.

Disclosure of Interest: None declared

DOI: 10.1136/annrheumdis-2017-eular.5579

\section{SAT0211 REAL WORLD DATA OF RITUXIMAB EFFECTIVENESS IN RHEUMATOID ARTHRITIS: DIFFERENCES BETWEEN BIOLOGIC-NAIVE PATIENTS AND PREVIOUSLY EXPOSED TO BIOLOGICS}

R.M. Ferreira, R. Fonseca, D.R. Gonçalves, F. Aguiar, T.M. Rocha, A. Bernardo, S. Pimenta, M. Bernardes, L. Costa. São João Hospital Centre, Porto, Portugal

Background: Rituximab is only approved for rheumatoid arthritis (RA) treatment in patients with an incomplete response or intolerance to others DMARDs, including TNF alfa inhibitors. It represents a significant advance in RA biologics arsenal due to its safety and efficacy profiles.

Objectives: To evaluate the effectiveness of rituximab in RA patients and to compare the response between first-line rituximab patients and those previously exposed to other biologics.

Methods: An observational retrospective study was conducted, including all the consecutive patients with diagnosis of RA under rituximab, followed at our Rheumatology department until December 2016. Demographic and clinical data were obtained by consulting the national database (Reuma.pt). DAS28 variations and EULAR response were measured at 6,12 and 18 months. Parametric and non parametric tests were used for statistics (SPSS 20.0).

Results: We included 63 RA patients ( $81 \%$ of women), with a mean (SD) age of 61 (10) years and a mean disease duration of $19(10)$ years; $86 \%$ rheumatoid factor positive and $87 \%$ anti- citrullinated peptide antibody (ACPA) positive. Bone erosions and extra-articular manifestations were present in $85,7 \%$ and $58,7 \%$ of the patients, respectively. At baseline, the mean DAS28 was $5.79(65 \%$ and $29 \%$ of patients with severe and moderate disease activity, respectively, and $6 \%$ in clinical remission). Thirty patients were treated with rituximab as first-line therapy and 33 patients were previously exposed to other biologics. Combination therapy 